\title{
Influencia de los Diseños de Agujas Endodónticas en la Irrigación de Conductos Radiculares
}

\author{
Influence of Endodontic Needle Designs on Root Canal Irrigation
}

\author{
Alessandra Baasch ${ }^{1}$; Karen Brisson-Suárez; Juan Miguel Koury²; \\ Víctor Talarico Leal Vieira ${ }^{1} \&$ Flavio Ferreira Alves ${ }^{1}$
}

\begin{abstract}
BAASCH, A.; BRISSON-SUÁREZ, K.; KOURY, J. M.; VIEIRA, V. T. L. \& ALVES, F. F. Influencia de los diseños de agujas endodónticas en la irrigación de conductos radiculares. Int. J. Odontostomat., 15(3):756-764, 2021.

RESUMEN: La irrigación del sistema de conductos radiculares con soluciones antibacterianas es considerada una parte esencial de la preparación químico-mecánica. Varios factores influyen en la eficacia de la irrigación del conducto radicular, incluyendo tamaño de la preparación apical y ensanchamiento, distancia de penetración de la aguja con respecto al ápice, flujo y volumen de sustancia de irrigante, dimensión de las agujas y la presencia de curvatura del conducto radicular. El tamaño de la preparación apical y la conicidad afectan el recambio del irrigante, el esfuerzo de corte en las paredes del conducto radicular y la presión en el foramen apical. La colocación de la punta de la aguja más cerca del ápice, permite un recambio de la solución más eficiente, resultando una mejor limpieza y desinfección del conducto radicular. La velocidad del irrigante, al lado de la aguja, en las paredes del conducto opuestas y frente a la salida de la aguja es dependiente del diseño de la aguja. El patrón de flujo y recambio del irrigante de las agujas con extremo abierto es diferente al de las agujas cerradas y las que logran mejor recambio de irrigante en la parte apical del conducto radicular también conducen a un aumento de la presión media en el foramen apical, lo que indica un mayor riesgo de extrusión del irrigante hacia el tejido periapical. El objetivo de esta revisión a la literatura, es evaluar los diferentes diseños de agujas y su influencia en la irrigación del sistema de conductos radiculares.
\end{abstract}

PALABRAS CLAVE: aguja irrigación, punta, diseño aguja irrigación.

\section{INTRODUCCIÓN}

La reducción de microorganismos dentro del sistema de conductos radiculares se logra mediante la limpieza químico-mecánica, siendo la irrigación un procedimiento esencial para el tratamiento de los conductos radiculares (Haapasalo et al., 2010). Una parte significativa del sistema de conductos radiculares no se puede alcanzar durante la instrumentación, grandes áreas de biopelícula bacteriana pueden permanecer dentro de los conductos radiculares, istmo, conductos accesorios y laterales (Peters, 2004; Ricucci et al., 2013) y restos de tejido pulpar, especialmente en el tercio apical (Siqueira Jr. et al., 2018; Zhao et al., 2019). La irrigación adecuada complementa la instrumentación mediante la combinación de los efectos químicos y mecánicos (Huang et al., 2008; Siqueira Jr. et al.).
El irrigante más utilizado hasta la actualidad es el hipoclorito de sodio. Las moléculas de hipoclorito de sodio son transportadas a lo largo del conducto radicular y sus variaciones anatómicas por el flujo masivo del irrigante y mediante el mecanismo de difusión (Pereira et al., 2021). Estudios recientes sugieren que tanto la clorhexidina como el hipoclorito de sodio pueden reducir las infecciones bacterianas sin diferencias significativas en la efectividad antimicrobiana, a pesar de sus diferencias en los mecanismos moleculares (Ruksakiet et al., 2020).

La solución de irrigación debe fluir por todo el sistema de conductos radiculares para tener contacto directo con los microorganismos, biopelícula, detritos, remanentes pulpares y proveer lubricación a los ins-

\footnotetext{
${ }^{1}$ Programa de Postgrado de Endodoncia, Universidad Grande Rio, Duque de Caxias, Rio de Janeiro, Brasil.

${ }^{2}$ Departamento de Endodoncia, Universidad Santa María, Caracas, Venezuela.
} 
trumentos. Es necesario el reemplazo frecuente de hipoclorito, ya que los componentes activos se consumen rápidamente cuando entran en contacto con bacterias, tejido pulpar o dentina (Moorer \& Wesselink, 1982). El efecto mecánico se produce a través de la fuerza cortante en la pared provocada por el irrigante que fluye sobre el biofilm (Pereira et al.). La penetración y el recambio del hipoclorito de sodio en el conducto radicular y las fuerzas de corte desarrolladas se ven muy afectadas por el flujo del irrigante (Boutsioukis et al., 2009).

Las agujas de irrigación de acero inoxidable cumplen con ciertas especificaciones ISO, concerniente tanto al diámetro externo como el interno y utilizan las unidades del sistema G (Boutsiuokis et al., 2007). Se han desarrollado varios diseños de puntas de agujas y técnicas de irrigación para mejorar las limitaciones de la desinfección químico-mecánica (Goldman et al., 1976, 1979; Moser \& Heuer, 1982; Kahn et al., 1995; Vinothkumar et al., 2007; Hülsmann et al., 2009). Comercialmente se pueden encontrar agujas de punta abierta, biseladas, con muesca, punta cerrada con salida lateral, punta cerrada con doble salida lateral y punta cerrada con múltiples salidas (Boutsioukis et al., 2010c).

Los objetivos principales del diseño de las agujas endodónticas de irrigación son maximizar la eficacia y la seguridad. La aguja de irrigación clásica tiene la salida en el extremo con una variedad de modificaciones en la punta de la aguja, tales como un bisel o una muesca. Las agujas con salida lateral tienen una punta redondeada y un puerto lateral que permiten que parte de la presión se transmita directamente y otra parte se ventile lateralmente. Estas deben doblarse fácilmente para que pueda seguir las curvaturas del conducto y permitir la administración de la solución de irrigación en todas las áreas del conducto radicular. Las agujas de calibre 27 a $30 \mathrm{G}$ son actualmente los tamaños de aguja más utilizados para la irrigación del conducto radicular porque son lo suficientemente pequeñas como para permitir el flujo de la solución en la mayoría de los conductos (Shen et al., 2010).

El diseño de la aguja puede solventar algunos de los problemas del proceso de irrigación, pero existe un conflicto entre el mejor diseño de la aguja para la penetración del irrigante y la óptima remoción de biofilm de las paredes del conducto radicular. Por lo tanto, el objetivo de esta revisión a la literatura, es evaluar los diferentes diseños de agujas y su influencia en la irrigación del sistema de conductos radiculares.

\section{DISEÑO DE LAS AGUJAS ENDODÓNTICAS E IN- FLUENCIA EN LA IRRIGACIÓN}

La efectividad del proceso de irrigación recae en la limpieza mecánica y la capacidad de la solución de irrigación de eliminar microorganismos y disolver tejido. Se ha demostrado que varios factores influyen en la eficacia de la limpieza química del conducto radicular, incluyendo tamaño de la preparación apical y ensanchamiento, distancia de penetración de la aguja con respecto al ápice, flujo y volumen de sustancia de irrigante, dimensión de las agujas y la presencia de curvatura del conducto radicular.

Tamaño de la preparación apical. La penetración del irrigante en la parte apical del sistema de conducto radicular es en gran parte dependiente del ensanchamiento apical adecuado durante la instrumentación (Ram, 1977; Chow, 1983; Kahn et al.). El tamaño de la preparación apical afecta el recambio del irrigante, el esfuerzo de corte en las paredes del conducto radicular y la presión en el foramen apical (Boutsioukis et al., 2010a).

Una irrigación efectiva no ocurre consistentemente a menos que se ensanche el conducto hasta un diámetro un \#25 (Boutsioukis et al., 2010a). Utilizando un instrumento hasta \#40 las agujas de irrigación de pequeño calibre penetran más en el conducto radicular (Ram), aunque se ha sugerido que con una técnica de preparación con ensanchamiento hasta un instrumento \#30 o \#35, el irrigante penetra en toda la profundidad de la instrumentación (Salzgeber \& Brilliant, 1977; Teplitsky et al., 1987) cuando es utilizada una aguja de 27G (Hsieh et al., 2007).

Al aumentar diámetro la preparación del conducto en la longitud de trabajo se produce un aumento en la limpieza del conducto radicular (Usman et al., 2004; Lee et al., 2019), aunque no se han mostrado ventajas al realizar la preparación a un instrumento mayor a \#60 (Falk \& Sedgley, 2005). Las preparaciones apicales amplias son beneficiosas para una irrigación exitosa en conductos radiculares instrumentados, pero al realizar la preparación hasta \#80 o superior causan turbulencias e irrigación incompleta del conducto radicular (Hsieh et al.).

Dependiendo del diámetro con que se ha ensanchado el conducto radicular se debe de utilizar la aguja adecuada que penetre lo suficientemente en el conducto para brindar una irrigación efectiva. Las agujas de menor calibre son más efectivas que las 
de mayor calibre (Chow). En dientes instrumentados hasta un instrumento \#25 las agujas de diámetros 23 G, 25 G y 27 G solo penetran dentro del conducto hasta $9 \mathrm{~mm}$ del ápice (Fig. 1-A) y los preparados hasta un instrumento \#30, las agujas con diámetros de $22 \mathrm{G}$ y $23 \mathrm{G}$ no llegan sino hasta $5-6 \mathrm{~mm}$ del ápice del conducto (Fig. 1-B), mientras que las de $27 \mathrm{G}$ se pueden colocar entre 1-2 mm del ápice (Fig. 1-C) (Kahn et al.) y las de $30 \mathrm{G}$ hasta el ápice (Fig. 1-D) (Chow). En una preparación hasta un instrumento \#40, la aguja de irrigación de $23 \mathrm{G}$ solo penetra hasta $6 \mathrm{~mm}$ del ápice (Fig. 1-E) mientras que una aguja de $30 \mathrm{G}$ puede llegar hasta $3 \mathrm{~mm}$ (Fig. 1-F) (AbouRass \& Piccinino, 1982). Un conducto radicular preparado con un instrumento \#45 una aguja de calibre $27 \mathrm{G}$, la cual tiene el mismo calibre que el instrumento \#45, puede ser colocada a $3 \mathrm{~mm}$ de ápice (Fig. 1G) (Wu \& Wesselink, 1995; Hsieh et al.). Cuando se aumenta la preparación del conducto radicular a \#50, tanto la aguja de calibre $27 \mathrm{G}$ como la de $25 \mathrm{G}$ y 23 $\mathrm{G}$ pueden ser colocadas a $3 \mathrm{~mm}$ del ápice de la raíz (Fig. 1-H). Por lo tanto, para los conductos radiculares más estrechos, es necesaria una aguja más fina insertada más cerca del ápice para una irrigación eficiente del conducto radicular (Hsieh et al.).

Mientras se aumenta el tamaño de la preparación, se debe tener precaución de no forzar el irrigante más allá del conducto radicular. Una instrumentación mayor que \#35 puede permitir la extrusión del irrigante más allá del ápice hacia los tejidos periapicales (Salzgeber \& Brilliant).
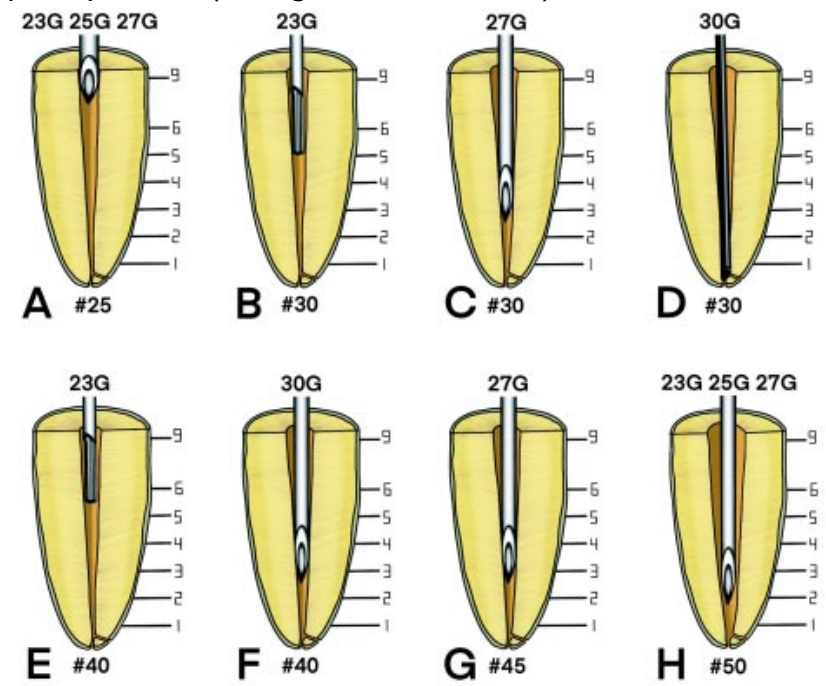

Fig. 1. Correlación de la preparación apical vs el calibre de la aguja. Hsieh et al., 2007 (A); Kahn et al., 1995 (B); Hsieh et al., 2007 (C); Chow, 1983 (D); Abou-Rass \& Piccinino, 1982 (E); Abou-Rass \& Piccinino, 1982 (F); Wu \& Wesselink, 1995, Hsieh et al., 2007 (G); Hsieh et al., 2007 (H).
Taper de la preparación. Un aumento en la conicidad del conducto radicular mejora el recambio del irrigante y la capacidad de corte de las paredes mientras reduce el riesgo de extrusión del irrigante. El flujo del irrigante en un conducto radicular con mínima conicidad y preparación apical amplia también mejora el recambio del irrigante. La fuerza de corte en la pared y se reduce el riesgo de extrusión del irrigante, comparado con los conductos radiculares con gran conicidad y preparación apical pequeña (Boutsioukis et al., 2010b).

La eliminación de debris mejora al aumentar la conicidad de la preparación. Sin embargo, no se han observado diferencias en la eliminación de debris entre preparaciones apicales diámetro \#20 y \#40 con una conicidad de .10. Esto puede ser debido a que al aumentar la conicidad de la preparación permite mayor penetración de la aguja de irrigación y mejora la limpieza de debris (Albrecht et al., 2004).

El paralelismo de las paredes en una preparación con conicidad .04 combinado con la leve curvatura presente en las diversas anatomías radiculares, pueden crear resistencia para una completa penetración de la aguja hasta la longitud de trabajo. En una preparación apical \#40 .04, una aguja de irrigación de $28 \mathrm{G}$ penetra hasta $2.25 \mathrm{~mm}$ de la longitud de trabajo (Albrecht et al.). En una preparación apical \#30 .02 la aguja de irrigación de 30G penetra solo hasta $3 \mathrm{~mm}$ de la longitud de trabajo. Sin embargo, una preparación apical \#60.02 ha mostrado resultados más favorables en comparación con preparaciones menos amplias en apical y mayor conicidad (Boutsioukis et al., 2010b).

Penetración de la aguja. La colocación de la punta de la aguja más cerca del ápice, permite un recambio de la solución más eficiente, resultando una mejor limpieza y desinfección del conducto radicular (Chow; Falk \& Sedgley; Bronnec et al., 2010). Existe influencia entre la penetración de la aguja de irrigación y la remoción de debris de tejidos duros. La colocación de una aguja a $1 \mathrm{~mm}$ de la longitud de trabajo, remueve 3 veces más cantidad de debris que si es colocada a $5 \mathrm{~mm}$ de la longitud de trabajo (Perez et al., 2017). Sin embargo, Usman et al. reportan que no hay ventajas con la inserción de la aguja más allá del $75 \%$ de la longitud de trabajo con respecto a la limpieza de los conductos radiculares.

Se ha observado poco intercambio de flujo y desplazamiento de partículas más allá de la punta de la aguja en conductos simulados (Chow; Sedgley 
et al., 2004). Una razón posible, es que el espacio entre la aguja y la pared del conducto no es lo suficientemente grande para el reemplazo del irrigante o la sustancia extracelular del biofilm, que actúa como una barrera física contra la penetración del irrigante de hipoclorito de sodio con su efecto químico ya agotado (Mohmmed et al., 2017).

A mayor penetración de la aguja proporciona mayor riesgo de extrusión de irrigante (Psimma et al., 2013). Las agujas con punta abierta presentan una extrusión significativamente mayor del irrigante que las agujas con punta cerrada. La extrusión del irrigante disminuye mientras la aguja se aleja de la longitud de trabajo o cuando el diámetro apical aumenta. Se presenta mayor riesgo de extrusión cuando la aguja se atasca en las paredes del conducto radicular, especialmente cuando se utiliza una aguja con punta abierta (Psimma et al.).

El uso de la aguja a una profundidad de penetración alejado de la longitud de trabajo puede ser una medida de seguridad contra la extrusión apical del irrigante, sin embargo, se deja una cantidad significativamente mayor de debris en variaciones anatómicas como istmos. La elección de una aguja adecuada colocada a un nivel apropiado es un paso importante para optimizar la calidad del proceso de irrigación (Perez et al.).

Velocidad de flujo y características de la aguja. El diseño de la punta de la aguja de irrigación influencia en el patrón de flujo, velocidad de flujo, y presión en la pared apical, todos parámetros importantes para la efectividad y riesgo de extrusión (Shen et al.). La distribución del flujo de la irrigación del conducto radicular puede verse afectada adversamente por agujas de diámetros amplios, por grandes distancias entre la punta de la aguja y el tope apical, y por conductos radiculares estrechos (Hsieh et al.).

Agujas de salida lateral con punta cerrada (Fig. 2D), en términos de eficiencia de reemplazo del irrigante, una tasa de flujo de 0.01-0.26 ml/s debería ser combinado con la colocación de la aguja de irrigación a $1 \mathrm{~mm}$ de la longitud de trabajo, con el fin de lograr un intercambio de irrigante aceptable. Tasas de flujo de $0.53-0.79 \mathrm{ml} / \mathrm{s}$ ofrecen una ventaja adicional de $0.5 \mathrm{~mm}$ pero no puede considerarse como condiciones clínicas promedio. Además, los valores aceptables pueden variar cuando se consideran diferentes anatomías de conductos o diferentes agujas. No obstante, cuanto mayor sea la velocidad del irrigante, más rápida y adecuada es la sustitución del mismo (Boutsioukis et al., 2009).

En las agujas de extremo cerrado (Figs. 2D-F) con una velocidad de irrigación de $0,1 \mathrm{ml} / \mathrm{s}$, no ocurre recambio de la solución, mientras que a una velocidad de $0,26 \mathrm{ml} / \mathrm{s}$ ocurre recambio de la solución a 1 $\mathrm{mm}$ de la punta. En las puntas de extremo abierto (Figs. 2A-C), el recambio del irrigante se extiende más allá de $2 \mathrm{~mm}$ apicalmente de la punta de la aguja (Boutsioukis et al., 2010c).

Un incremento en el tamaño y conicidad de la preparación mejora el recambio de la solución (Chow; Huang et al.; Boutsioukis et al., 2010c). Es importante tener en cuenta que la presencia de una salida lateral en la aguja de irrigación resulta en unareducción de la presión apical de $17 \%-19 \%$, mientras que una aguja con punta biselada (Fig. 2A) aumenta la presión apical en comparación con puntas con muescas (Fig. 2C) en $7 \%-10 \%$. Este aumento es debido a que el bisel extiende la distancia sobre la cual el irrigante puede mantener su alta velocidad de flujo presente en el lumen de la aguja. Cerrar la punta de la aguja con salida lateral conduce a una disminución de 2.5 a 3 veces la presión apical, apoyando las afirmaciones de riesgo reducido de extrusión apical para agujas con extremos cerrados (Shen et al.).

De las agujas con extremo abierto, la aguja plana (Fig. 2B) y biselada presentan flujos similares de irrigante a alta velocidad en el conducto radicular. Para

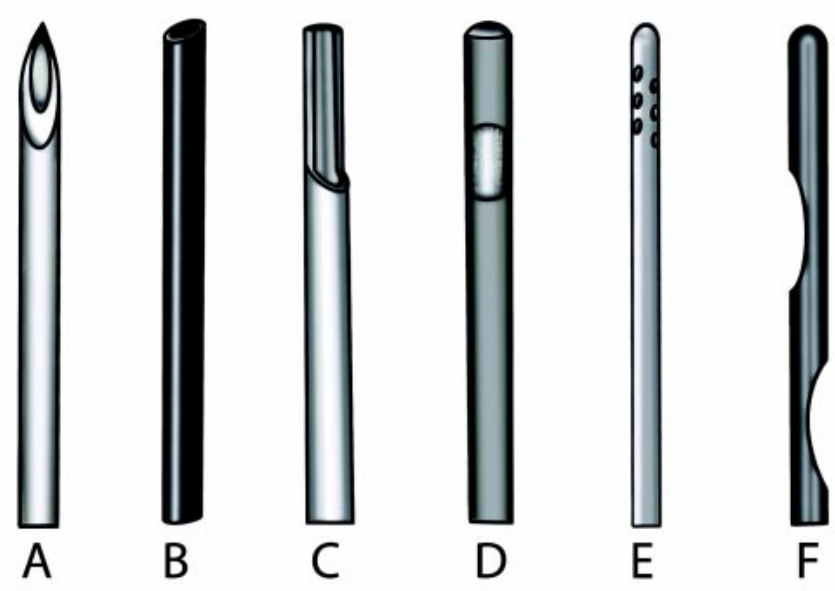

Fig. 2. Representación de los tipos de agujas endodónticas. Biselada (A); plana (B); muesca (C); extremo cerrado con salida lateral (D); extremo cerrado con múltiples salidas $(E)$; extremo cerrado con doble salida lateral (F). 
la aguja con muescas, las velocidades en el flujo son ligeramente menores. El patrón de flujo de las agujas de punta cerrada es diferente en comparación con las agujas de punta abierta. El flujo es más dirigido a la pared del conducto radicular en lugar del ápice. En las agujas con salida lateral y doble salida lateral se presenta un patrón de flujo con un torrente de irrigante formado en la salida, el proximal a la punta para la doble salida lateral (Fig. 2F), y dirigido hacia el ápice. El irrigante sigue una vía curva alrededor de la punta. El flujo de la salida lateral presenta solo una influencia menor en el patrón de flujo (Boutsioukis et al., 2010c).

La velocidad del irrigante, al lado de la aguja, en las paredes del conducto opuestas y frente a la salida de la aguja es dependiente del diseño de la aguja. Para la aguja con muesca y biselada colocada a $3 \mathrm{~mm}$ del ápice, la velocidad de flujo fue ligeramente más rápida en el lado opuesto, probablemente como resultado de la permeabilidad asimétrica entre los 2 lados creada por la muesca / bisel. Cuando es colocada a $5 \mathrm{~mm}$ del ápice, no se identifican diferencias entre la velocidad del flujo en las paredes opuestas para estas agujas. Para las agujas con salida lateral, el flujo en el lado opuesto a la salida lateral es muy bajo, acercándose a cero para las agujas con salida lateral y punta cerrada (Shen et al.).

Existe una correlación inversa entre el $\mathrm{G}$ de las agujas de endodoncia y la tasa de flujo de irrigación. La tasa de flujo clínico es de $0.27 \mathrm{ml}^{-1}$ para agujas de 26G, $0.19 \mathrm{ml}^{-1}$ para 27G, y $0.09 \mathrm{ml}^{-1}$ para agujas de $30 \mathrm{G}$. Aunque las agujas de $26 \mathrm{G}$ y $27 \mathrm{G}$ pueden proporcionar una mayor tasa de flujo, dentro del conducto radicular el diámetro externo de estas agujas perjudica la profundidad de la penetración. Por otro lado, la aguja de $30 \mathrm{G}$ puede colocarse tan apical clínicamente como sea posible sin atascarse. Sin embargo, la aguja de 30G presenta el menor flujo, lo cual puede influenciar la dinámica de reemplazo del fluido del irrigante en el tercio apical del conducto radicular (Gopikrishna et al., 2016).

La fuerza de corte en la pared del conducto influye en el desprendimiento mecánico de debris, restos de tejido, microorganismo aislados, y biopelícula. Aunque no existen datos cuantitativos sobre la mínima fuerza de corte requerida, la distribución de la fuerza cortante a lo largo de la pared del conducto proporciona una indicación de la eficacia de desbridamiento de cada tipo de aguja. Debe enfatizarse que la ruptura o desprendimiento de biopelículas o de debris no pueden asegurar su remoción a menos que haya un flujo inverso del irrigante favorable para llevarlos hacia el orificio del conducto (Boutsioukis et al., 2010c).

La fuerza de corte desarrollada por las agujas de salida lateral y doble salida lateral es significativamente más alto en la pared que se encuentra en contacto con la salida (Boutsioukis et al., 2010c). La segunda salida de la aguja con doble salida lateral afecta ligeramente el rendimiento general y no parece proporcionar ninguna ventaja (Vinothkumar et al.). Por lo tanto, la superficie del conducto radicular que se encuentra de frente a la salida de la aguja queda más limpia que la del lado opuesto, lo cual tiene una trascendencia significativa para el diseño de la aguja (Huang et al.).

El área de alta fuerza de corte se presenta en la parte apical del conducto, relativamente cerca de la punta de la aguja, área donde el desprendimiento de biopelicula y debris se espera que sea más eficiente (Huang et al.; Boutsioukis et al., 2010c). Las agujas que logran mejor recambio de irrigante en la parte apical del conducto radicular también conducen a un aumento de la presión media en el foramen apical, lo que indica un mayor riesgo de extrusión del irrigante hacia el tejido periapical. Desde un punto de vista clínico, la prevención de la extrusión debe preceder al requisito de un recambio adecuado de irrigante y fuerza cortante (Boutsioukis et al., 2010c; Hüllsmann et al.).

Observaciones en el flujo del irrigante dentro de los conductos han demostrado que la aguja de salida lateral puede presentar riesgos si el diámetro de la aguja es pequeño y este alcanza la longitud completa del conducto, ya que esto permite que los irrigantes fluyan hacia el área periapical. Con una aguja estándar, el fluido no pasa más allá de la punta de la aguja cuando se emplea una presión mínima en la irrigación. Mientras aumenta el tamaño del conducto, no ocurre la extrusión más allá de la punta. Sin embargo, si la punta se atasca en el conducto, es forzado fuera de la punta de la aguja, y la presión aplicada a la jeringa es directamente proporcional a la distancia que el fluido fluye fuera de la punta de la aguja (Devi \& Abbott, 2012).

La aguja de punta cerrada con salida lateral también ha mostrado que ocurre extrusión del irrigante a través del foramen apical cuando se utiliza una aguja de diámetro pequeño y se aplica más presión a la jeringa. Por lo tanto, este tipo de aguja puede ser de alto riesgo si se utiliza inapropiadamente (Devi \& Abbott). 
Volumen del irrigante. En cuanto al volumen del irrigante que debe utilizarse mientras se aumenta el volumen, se reduce significativamente la carga bacteriana; evidenciando que al irrigar con $6 \mathrm{~mL}$ de hipoclorito de sodio, se remueve el $92 \%$ de los microorganismos (Sedgley et al.).

Un alto volumen de hipoclorito de sodio, $13 \mathrm{ml}$, tiene una influencia positiva en la desinfección de los conductos radiculares durante la preparación químico mecánica en vez de la concentración (Gazzaneo et al., 2019), al igual que el flujo del irrigante (Pereira et al.).

Dimensión de la aguja. El tamaño de la aguja determina en parte la profundidad de colocación de la misma, ya que una aguja de calibre $21 \mathrm{G}$ puede alcanzar el ápice de conductos de \#80, una aguja de $23 \mathrm{G}$ a $\# 50$, una de $25 \mathrm{G}$ a \#45 y una aguja de $30 \mathrm{G}$ a \#20 (Chow).

Es importante el conocimiento exacto del diámetro de la punta de la aguja para la selección apropiada el tratamiento de conducto radicular. A pesar de la técnica de irrigación que se emplee, la profundidad de penetración del irrigante juega un papel importante, por lo cual se han realizado diversas evaluaciones y se han diseñado diferentes tipos de agujas. Las agujas de irrigación son fabricadas en acero inoxidable y cumplen con las especificaciones ISO 9626:1991 and 9626:1991/Amd 1:2001.Las agujas de NiTi no son incluidas en las especificaciones, y exceden los límites externos de las especificaciones ISO (Boutsioukis et al., 2007).

Un hallazgo común independientemente del tipo de aguja y fabricante es la presencia de numerosas irregularidades en la superficie interna. Estas irregularidades ocupan un área pequeña pero importante del lumen de la aguja y puede alterar el flujo del irrigante. Aunque la norma ISO (Boutsioukis et al., 2007) se refiere al acabado de la superficie externa, no existe ninguna referencia sobre la morfología de la superficie interna. El diámetro externo tanto de la aguja como de la lima para minimizar el riesgo de que la aguja se atasque en el conducto radicular. Esta correlación ayuda a la selección del tamaño de la aguja apropiada, debido a la familiaridad con la estandarización de los instrumentos de endodoncia (Zinelis et al., 2002).

Las agujas de $25 \mathrm{G}$ fueron comúnmente utilizadas para irrigación en endodoncia hace unos años, fueron reemplazadas primero por agujas de $27 \mathrm{G}$, ahora por $30 \mathrm{G}$ y hasta agujas de $31 \mathrm{G}$ están tomando ventaja en la rutina de la irrigación. Como $27 \mathrm{G}$ corresponde a estándares internacionales de $0.42 \mathrm{~mm}$ y $30 \mathrm{G}$ a 0.31 $\mathrm{mm}$ de diámetro, se prefieren las agujas de menor calibre (Haapasalo et al.).

Mientras mayor es el gauge de la aguja, es decir menor diámetro, a mayor longitud se puede introducir en el conducto radicular (Devi \& Abbott).

Conductos curvos. En la presencia de conductos curvos, al aumentar la curvatura del conducto radicular se impide el flujo del irrigante, así reduciendo su capacidad de limpieza y disminuyendo su eficacia mecánica, por lo tanto aumentando el tamaño de la preparación apical hasta \#46/0.04 se mejora significativamente la eficacia de la irrigación (Nguy \& Sedgley, 2006). La curvatura moderada del conducto radicular no tiene un efecto significativo en la extrusión del irrigante (Psimma et al.).

\section{DISCUSIÓN}

Los diversos diseños de las puntas de las agujas de irrigación han sido utilizados en modelos in vitro, mediante diferentes procedimientos, para evaluar su influencia en la efectividad de la irrigación en la porción apical del conducto radicular (Chow; Hsieh et al.; Boutsioukis et al., 2010c). Se le ha dado gran importancia al efecto del flujo y presión del irrigante en el conducto (Boutsioukis et al., 2010c), dominado por factores como la preparación del conducto y diámetro de la aguja (Boutsioukis et al., 2010a) los cuales tiene gran influencia en la penetración de la aguja dentro del sistema de conductos radiculares (Psimma et al.).

La penetración del irrigante en el sistema de conducto radicular preparado es un factor importante y es una función del diámetro de la aguja de irrigación en relación al tamaño de la preparación (Zehnder, 2006). Estudios previos sugieren que la preparación apical del conducto radicular debe realizarse hasta mínimo instrumentos \#30-40 (Ram; Salzgeber \& Brilliant; Teplitsky et al.) (Tabla I), y conicidad hasta al menos .04 para lograr la penetración de la aguja hasta el tercio apical (Nguy \& Sedgley).

Anteriormente eran muy utilizadas las agujas de punta biselada, a las cuales se les realizaban desgastes en el extremo para evitar que se atascaran en las 
Tabla I. Preparación Apical Mínima y Máxima Recomendada

\begin{tabular}{lccc}
\hline \multicolumn{1}{c}{ Autor } & Año & $\begin{array}{c}\text { Preparación } \\
\text { Apical Mínima }\end{array}$ & $\begin{array}{c}\text { Preparación } \\
\text { Apical Máxima }\end{array}$ \\
\hline Ram & 1977 & $\# 40$ & \\
Salzgeber \& Brilliant & 1977 & $\# 30$ & $\# 35$ \\
Teplitsky et al. & 1987 & $\# 30$ & \\
Falk \& Sedgley & 2005 & & $\# 60$ \\
Hsieh et al. & 2007 & $\# 30$ & $\# 80$ \\
Boutsioukis et al. & 2010 & $\# 25$ &
\end{tabular}

paredes del conducto. Mediante la simulación de partículas en conjunto con la utilización de tinta, se ha podido observar que la irrigación con agujas biseladas de $23 G$ y $25 G$ es más efectiva mientras aumenta la preparación del conducto debido a que se logra una mayor inserción de la aguja de irrigación en el conducto radicular (Chow). Mediante un sistema de análisis de imágenes térmicas, también se ha podido observar que el flujo de irrigación alcanza al ápice en conductos amplios \#30 y \#40 cuando una aguja 27G es colocada cerca del ápice (Hsieh et al.).

Un estudio de Dinámica de Fluidos Computacional en simulaciones de conductos radiculares preparados hasta \#45, evaluó agujas de $30 \mathrm{G}$ con extremo abierto y extremo cerrado. Las agujas con extremo abierto, las biseladas y de punta plana, presentan corrientes similares del irrigante de alta velocidad en el conducto radicular y mayor recambio del irrigante. Además la corriente que se forma más apicalmente de la punta plana se extiende más lejos de la punta a medida que aumenta el tamaño de la preparación. Mientras que en las de punta cerrada la velocidad de la corriente es menor y el recambio del irrigante ocurre sólo hasta $0.75 \mathrm{~mm}$ apicalmente de la punta de la aguja (Boutsioukis et al., 2010a,c).

La evaluación de la eficacia del sistema de irrigación con una aguja de $27 \mathrm{G}$ con muesca, mediante la presencia o ausencia de tinta remanente en el conducto radicular luego de la irrigación, es altamente efectiva en conductos instrumentados hasta \#30 y \#35 colocada a 1-2 $\mathrm{mm}$ del foramen apical. El fluido a través del lumen de estas agujas, crea turbulencia alrededor y más allá de la punta de la aguja (Kahn et al.).

Tomando en cuenta la profundidad de penetración de la aguja y la velocidad del flujo del irrigante (Fig. 1), se recomienda que las agujas de extremo cerrado deben colocarse a $1 \mathrm{~mm}$ de la longitud de trabajo, mientras que las agujas de extremo abiertos deben ser colocadas de 2 a $3 \mathrm{~mm}$ de la longitud de tra- bajo (Boutsioukis et al., 2010c). Cuando una aguja de $30 \mathrm{G}$ es utilizada, la preparación apical debe realizarse hasta diámetros \#35-40 para asegurar una limpieza adecuada de la zona apical (Zehnder). Un incremento en el tamaño y conicidad de la preparación mejora el recambio de la solución (Chow; Huang et al.; Boutsioukis et al., 2010a,b).

Para mejorar la seguridad de la irrigación y prevenir la extrusión apical del irrigante, se recomiendan usar agujas con salida lateral con punta cerrada y segura (Hülsmann et al.), a pesar de que se ha demostrado que las agujas de salida lateral generan menor limpieza en la pared contraria a la salida lateral de la aguja (Huang et al.).

Vinothkumar et al. sugiere que las agujas con punta de seguridad y una salida lateral son eficientes en la remoción mecanica de bacterias en conductos radiculares instrumentados, mientras que Boutsiukis et al. (c), 2010 ha sugerido que ocurre más recambio de irrigante en la zona delante de las agujas abiertas pero también una presión apical más alta.

Además de la importancia del $\mathrm{G}$ y penetración de la aguja, se ha sugerido que existen otros factores más importantes en la eficacia mecanica de la irrigación, como el volumen del irrigante. Mientras mayor sea el volumen de la solución química desinfectante, $13 \mathrm{ml}$ de hipoclorito de sodio, (Gazzaneo et al.), menor cantidad de microorganismos remanentes en el sistema de conductos radiculares (Nguy \& Sedgley).

Debido a las diversas anatomías de los conductos radiculares, una aguja debería doblarse fácilmente para permitir que siga la curvatura del conducto y permita llevar el irrigante a todas las áreas del conducto radicular. En la actualidad, las agujas de 27-30 G son los calibres de agujas más utilizadas para la irrigación de conductos radiculares ya que son suficientemente pequeñas para permitir el retorno de la solución en la mayoría de los conductos (Shen et al.). 


\section{CONCLUSIÓN}

El patrón de flujo de las agujas abiertas es diferente al de las agujas cerradas resultando en más recambio de irrigante en la zona más apical a la punta de las agujas abiertas pero también una presión apical más alta. Al hacer mayor recomendación en la literatura del uso de agujas de punta cerrada con ventana lateral, se sacrifica la eficacia de desinfección del conducto radicular por seguridad de riesgo de extrusión del irrigante a los tejidos periapicales. Al tener conocimiento de los diferentes diseños de agujas y el patrón de flujo correspondiente del irrigante, se puede hacer buen uso de las agujas con extremos abiertos sin riesgo de extrusión del irrigante a los tejidos periapicales.

\section{AGRADECIMIENTOS}

Este estudio fue parcialmente financiado por el Consejo Nacional de desarrollo Científico y Tecnológico (CNPq) y la Fundaçión de Amparo e Investigación del Estado de Rio de Janeiro (FAPERJ).

BAASCH, A.; BRISSON-SUÁREZ, K.; KOURY, J. M.; VIEIRA, V. T. L. \& ALVES, F. F. Influence of endodontic needle designs on root canal irrigation. Int. J. Odontostomat., 15(3):756-764, 2021.

ABSTRACT: Root canal irrigation with antibacterial solutions is considered an essential part of the chemical-mechanical preparation. Several factors influence the efficacy of root canal irrigation, including apical preparation size and taper, needle penetration distance from the apex, flow and volume of irrigant substance, dimension of the needles and the presence of curvature of the root canal. The apical preparation size and the taper affect irrigant replacement, shear stress on the root canal walls, and pressure on the apical foramen. Placing the tip of the needle closer to the apex, allows more efficient solution exchange, resulting in better cleaning and disinfection of the root canal. The velocity of the irrigant, next to the needle, on the opposite canal walls and in front of the needle outlet is dependent on the design of the needle. The flow and irrigant replacement pattern of open ended needles is different from needles with closed tip. The needles that achieve better irrigant exchange in the apical part of the root canal also lead to an increase in mean pressure in the apical foramen which indicates a greater risk of extrusion of the irrigant into the periapical tissue. The objective of this literature review is to evaluate the different needle designs and their influence on the root canal system irrigation.

KEY WORDS: needle irrigation, tip, needle irrigation design.

\section{REFERENCIAS BIBLIOGRÁFICAS}

Abou-Rass, M. \& Piccinino, M. V. The effectiveness of four clinical irrigation methods on the removal of root canal debris. Oral Surg. Oral Med. Oral Pathol., 54(3):323-8, 1982.

Albrecht, L. J.; Baumgartner, J. C. \& Marshall, J. G. Evaluation of apical debris removal using various sizes and tapers of ProFile GT files. J. Endod., 30(6):425-8, 2004.

Boutsioukis, C.; Gogos, C.; Verhaagen, B.; Versluis, M.; Kastrinakis E. \& Van der Sluis, L. W. M. The effect of apical preparation size on irrigant flow in root canals evaluated using an unsteady Computational Fluid Dynamics model. Int. Endod. J., 43(10):87481, 2010a.

Boutsioukis, C.; Gogos, C.; Verhaagen, B.; Versluis, M.; Kastrinakis, E. \& Van der Sluis, L. W. M. The effect of root canal taper on the irrigant flow: evaluation using an unsteady Computational Fluid Dynamics model. Int. Endod. J., 43(10):909-16, 2010b.

Boutsioukis, C.; Lambrianidis, T. \& Kastrinakis, E. Irrigant flow within a prepared root canal using various flow rates: a Computational Fluid Dynamics study. Int. Endod. J., 42(2):144-55, 2009.

Boutsioukis, C.; Lambrianidis, T. \& Vasiliadis, L. Clinical relevance of standardization of endodontic irrigation needle dimensions according to the ISO 9,626:1991 and 9,626:1991/Amd 1:2001 specification. Int. Endod. J., 40(9):700-6, 2007.

Boutsioukis, C.; Verhaagen, B.; Versluis, M.; Kastrinakis, E.; Wesselink, P. R. \& van der Sluis, L. W. M. Evaluation of irrigant flow in the root canal using different needle types by an unsteady computational fluid dynamics model. J. Endod., 36(5):875-9, 2010c.

Bronnec, F.; Bouillaguet, S. \& Machtou, P. Ex vivo assessment of irrigant penetration and renewal during the final irrigation regimen. Int. Endod. J., 43(8):663-72, 2010.

Chow, T. W. Mechanical effectiveness of root canal irrigation. J. Endod., (11)9:475-9, 1983.

Devi, A. A. \& Abbott, P. V. Comparison of the flow characteristics of irrigants with standard and Max-i-Probe needles. Aust. Endod. J., 38(2):50-4, 2012.

Falk, K. W. \& Sedgley, C. M. The influence of preparation size on the mechanical efficacy of root canal irrigation in vitro. J. Endod., 31(10):742-5, 2005.

Gazzaneo, I.; Vieira, G.; Pérez, A. R.; Alves, F. R. F.; Gonçalves, L. S.; Mdala, I.; Siqueira Jr., J. F. \& Rôças I. N. Root canal disinfection by single- and multiple-instrument systems: effects of sodium hypochlorite volume, concentration, and retention time. J. Endod., 45(6):736-41, 2019.

Goldman, L. B.; Goldman, M.; Kronman, J. H. \& Lin, P. S. Scanning electron microscope study of a new irrigation method in endodontic treatment. Oral Surg. Oral Med. Oral Pathol., 48(1):79-83, 1979.

Goldman, M.; Kronman, J. H.; Goldman, L. B.; Clausen, H. \& Grady, J. New method of irrigation during endodontic treatment. J. Endod., 2(9):257-60, 1976. 
Gopikrishna, V.; Sibi, S.; Archana, D.; Kumar, A. R. P. \& Narayanan, L. An in vivo assessment of the influence of needle gauges on endodontic irrigation flow rate. J. Conserv. Dent., 19(2):189-93, 2016.

Haapasalo, M.; Shen, Y.; Qian, W. \& Gao, Y. Irrigation in endodontics. Dent. Clin. North Am., 54(2):291-312, 2010.

Hsieh, Y. D.; Gau, C. H.; Wu, S. F. K.; Shen, E. C.; Hsu, P. W. \& Fu, E. Dynamic recording of irrigating fluid distribution in root canals using thermal image analysis. Int. Endod. J., 40(1):11-7, 2007.

Huang, T. Y.; Gulabivala, K. \& Ng, Y. L. A bio-molecular film ex-vivo model to evaluate the influence of canal dimensions and irrigation variables on the efficacy of irrigation. Int. Endod. J., 41(1):60-71, 2008.

Hülsmann, M.; Rödig, T. \& Nordmeyer, S. Complications during root canal irrigation. Endod. Top., 16(1):27-63, 2009.

Kahn, F. H.; Rosenberg, P. A. \& Gliksberg, J. An in vitro evaluation of the irrigating characteristics of ultrasonic and subsonic handpieces and irrigating needles and probes. J. Endod., 21(5):277-80, 1995

Lee, O. Y. S.; Khan, K.; Li, K. Y.; Shetty, H.; Abiad, R. S.; Cheung, G. S. P. \& Neelakantan, P. Influence of apical preparation size and irrigation technique on root canal debridement: a histological analysis of round and oval root canals. Int. Endod. J., 52(9):136676, 2019.

Mohmmed, S. A.; Vianna, M. E.; Penny, M. R.; Hilton, S. T. \& Knowles, J. C. The effect of sodium hypochlorite concentration and irrigation needle extension on biofilm removal from a simulated root canal model. Aust. Endod. J., 43(3):102-9, 2017.

Moorer, W. F. \& Wesselink, P. R. Factors promoting the tissue dissolving capability of sodium hypochlorite. Int. Endod. J., 15(4):187-96, 1982.

Moser, J. B. \& Heuer, M. A. Forces and efficacy in endodontic irrigation systems. Oral Surg. Oral Med. Oral Pathol., 53(4):425-8, 1982.

Nguy, D. \& Sedgley, C. The influence of canal curvature on the mechanical efficacy of root canal irrigation in vitro using real-time imaging of bioluminescent bacteria. J. Endod., 32(11):1077-80, 2006.

Pereira, T. C.; Boutsioukis, C.; Dijkstra, R. J. B.; Petridis, X.; Versluis, M.; de Andrade, F. B.; van de Meer, W. J.; Sharma, P. K.; van der Sluis, L. W. M. \& So, M. V. R. Biofilm removal from a simulated isthmus and lateral canal during syringe irrigation at various flow rates: a combined experimental and Computational Fluid Dynamics approach. Int. Endod. J., 54(3):427-38, 2021.

Perez, R.; Neves, A. A.; Belladonna, F. G.; Silva, E. J. N. L.; Souza, E. M.; Fidel, S.; Versiani, M. A.; Lima, I.; Carvalho, C. \& De-Deus G. Impact of needle insertion depth on the removal of hard-tissue debris. Int. Endod. J., 50(6):560-8, 2017.

Peters, O. A. Current challenges and concepts in the preparation of root canal systems: a review. J. Endod., 30(8):559-67, 2004.

Psimma, Z.; Boutsioukis, C.; Kastrinakis, E. \& Vasiliadis L. Effect of needle insertion depth and root canal curvature on irrigant extrusion ex vivo. J. Endod., 39(4):521-4, 2013.

Ram, Z. Effectiveness of root canal irrigation. Oral Surg. Oral Med. Oral Pathol., 44(2):306-12, 1977.

Ricucci, D.; Loghin, S. \& Siqueira Jr., J. F. Exuberant Biofilm infection in a lateral canal as the cause of short-term endodontic treatment failure: report of a case. J. Endod., 39(5):712-8, 2013.

Ruksakiet, K.; Hanák, L.; Farkas, N.; Hegyi, P.; Sadaeng, W.; Czumbel, L. M.; Sang-Ngoen, T.; Garami, A.; Mikó, A.; Varga, G.; et al. Antimicrobial efficacy of chlorhexidine and sodium hypochlorite in root canal disinfection: a systematic review and meta-analysis of randomized controlled trials. J. Endod., 46(8):1032-1041.e7, 2020

Salzgeber, R. M. \& Brilliant, J. D. An in vivo evaluation of the penetration of an irrigating solution in root canals. J. Endod., 3(10):394-8, 1977.
Sedgley, C.; Applegate, B.; Nagel, A. \& Hall, D. Real-time imaging and quantification of bioluminescent bacteria in root canals in vitro. J. Endod., 30(12):893-8, 2004.

Shen, Y.; Gao, Y.; Qian, W.; Ruse, N. D.; Zhou, X.; Wu, H. \& Haapasalo, M. Three-dimensional numeric simulation of root canal irrigant flow with different irrigation needles. J. Endod., 36(5):884-9, 2010.

Siqueira Jr., J. F.; Pérez, A. R.; Marceliano-Alves, M. F.; Provenzano, J. C.; Silva, S. G.; Pires, F. R.; Vieira, G. S. C.; Rôças, I. N. \& Alves F. R. F. What happens to unprepared root canal walls: a correlative analysis using micro-computed tomography and histology/scanning electron microscopy. Int. Endod. J., 51(5):5018, 2018.

Teplitsky, P. E.; Chenail, B. L.; Mack, B. \& Machnee, C. H. Endodontic irrigation--a comparison of endosonic and syringe delivery systems. Int. Endod J., 20(5):233-41, 1987.

Usman, N.; Baumgartner, J. C. \& Marshall, J. G. Influence of instrument size on root canal debridement. J. Endod., 30(2):1102, 2004.

Vinothkumar, T. S.; Kavitha, S.; Lakshminarayanan, L.; Gomathi, N. S. \& Kumar, V. Influence of irrigating needle-tip designs in removing bacteria inoculated into instrumented root canals measured using single-tube luminometer. J. Endod., 33(6):7468, 2007.

Wu, M. K. \& Wesselink, P. R. Efficacy of three techniques in cleaning the apical portion of curved root canals. Oral Surg. Oral Med. Oral Pathol. Oral Radiol. Endod., 79(4):492-6, 1995.

Zehnder, M. Root canal irrigants. J. Endod., 32(5):389-98, 2006.

Zhao, Y.; Fan, W.; Xu, T.; Tay, F. R.; Gutmann, J. L. \& Fan B. Evaluation of several instrumentation techniques and irrigation methods on the percentage of untouched canal wall and accumulated dentine debris in C-shaped canals. Int. Endod. J., 52:1354-1365, 2019.

Zinelis, S.; Magnissalis, E. A.; Margelos, J. \& Lambrianidis, T. Clinical relevance of standardization of endodontic files dimensions according to the ISO 3630-1 specification. J. Endod., 28(5):36770,2002

Dirección para correspondencia:

Alessandra Baasch

Programa de Postgrado de Endodoncia

Universidad Grande Rio

Duque de Caxias

Rio de Janeiro

BRASIL

E-mail: endobaasch@gmail.com 